\title{
Effect of Treadmill Training With Eyes Open and Closed on Knee Proprioception, Functional Balance and Mobility in Children With Spastic Diplegia
}

\author{
Samah Attia El Shemy, PhD \\ Department of Physical Therapy for Pediatrics, Faculty of Physical Therapy, Cairo University, Giza, Egypt
}

\begin{abstract}
Objective To investigate the effect of treadmill training with eyes open (TEO) and closed (TEC) on the knee joint position sense (JPS), functional balance and mobility in children with spastic diplegia.

Methods Forty-five children with spastic diplegia aged 11-13 years participated in this study. They were randomly assigned to three groups of equal number. The control group (CON) underwent designed physical therapy program whereas, the study groups (TEO and TEC) underwent the same program, in addition to treadmill gait training with eyes open and closed, respectively. Outcome measures were the degree of knee joint position error, functional balance and mobility. Measurements were taken before and after 12 weeks of intervention.

Results After training, the three groups showed statistically significant improvement in all measured outcomes, compared to the baseline with non-significant change in the knee JPS in the CON group. When comparing posttreatment results, the TEC group showed greater significant improvement in all measured outcomes, than the TEO and CON groups.

Conclusion Treadmill training with eyes open and closed is effective in rehabilitation of children with diplegia, but blocked vision treadmill training has more beneficial effect.
\end{abstract}

Keywords Treadmill, Vision, Proprioception, Postural control, Rehabilitation, Cerebral palsy

\section{INTRODUCTION}

Appropriate postural control requires integration of sensory and motor systems. The network of the vestibu- lar, proprioceptive and visual systems is crucial for typical motor responses, balance control, and mobility [1]. Participation of each sensory framework varies according to environmental circumstances [2]. Considering loco-

Received April 6, 2018; Accepted June 15, 2018

Corresponding author: Samah Attia El Shemy

Department of Physical Therapy for Pediatrics, Faculty of Physical Therapy, Cairo University, 7 Ahmed Elzayat Street, Dokki, Giza, 12612 Egypt. Tel: +20-01005014313, Fax: +20-237617692, E-mail: samah_elshemy@yahoo.com

ORCID: Samah Attia El Shemy (http://orcid.org/0000-0002-7599-5212).

(c) This is an open-access article distributed under the terms of the Creative Commons Attribution Non-Commercial License (http://creativecommons.org/ licenses/by-nc/4.0) which permits unrestricted noncommercial use, distribution, and reproduction in any medium, provided the original work is properly cited. Copyright $\odot 2018$ by Korean Academy of Rehabilitation Medicine 
motion, the visual system recognizes feedback or feedforward information concerning alterations in the walking pathway, enhancing postural adjustment according to the environment [3]. In visual deprivation, posture is modified through sensory inputs from vestibular, proprioceptive, and tactile frameworks [4]. Therefore, in the event of lessened contribution of one sensory framework, other frameworks may be required to expand their involvement to assure achievement of a task [5].

Regarding all the sensory inputs, proprioception is viewed as the one most firmly connected to locomotor activity. Joint position sense (JPS) recognizes movement of joints, and assumes an essential role in gait and postural control [6]. Proprioception deficits in cerebral palsy (CP) are most likely due to central nervous system lesions, which impact proprioceptive inputs coming from muscle spindles, Golgi tendon organs, and sensory innervations of joints and skin to the cortex [7]. Additionally, spasticity causes changes in muscle fibers that may impede the JPS influencing the connection between muscles and joints and also affecting muscle spindle sensitivity [8].

Spastic diplegia is one of the most common types of $\mathrm{CP}$ in which lower limbs are affected more than upper limbs. Children with diplegic CP encounter difficulties in sensory processing and integration which influence the achievement of mature postural control [9]. Consequently, they have balance impairments in altered sensory environments, and tend to depend excessively on visual input to maintain posture and to position their limbs during gait, which may indicate proprioception deficits [10]. They also experience difficulty with planning of motor responses, in response to support surface perturbations due to delayed onset of muscle activation. Moreover, these children demonstrate deficiency in anticipatory postural adjustments while standing and walking [11].

Treadmill training is assumed to modify postural control in children with $\mathrm{CP}$, by permitting multiple repetitions of steps in rhythmic pattern during the gait cycle. Additionally, it adjusts control between agonist and antagonist muscles, leading to enhanced walking speed, and static and functional balance [12,13]. No studies have addressed the possible effect of treadmill training with eyes closed on knee joint proprioception, functional balance and mobility in children with spastic diplegia.
Moreover, there was significant error in the knee JPS in children with spastic diplegia, when compared with typically developing children [14]. Accordingly, more studies have to be conducted on the correction of proprioceptive error in children with diplegia. In the same context, frequent falling is considered one of the widespread problems in children with $\mathrm{CP}$ who are able to walk alone [15]. Consequently, disturbed balance in a functional background is a noteworthy complication to be considered in rehabilitation programs. Therefore, the purpose of this study was to investigate the effect of treadmill gait training with eyes open (TEO) and closed (TEC) on recognition of knee JPS, functional balance and mobility in children with spastic diplegia, and to determine which training protocol is more effective.

\section{MATERIALS AND METHODS}

\section{Participants}

Fifty spastic diplegic CP children of both sexes were assessed for eligibility of this study. They were recruited from the pediatric physical therapy outpatient clinic of the Faculty of Physical Therapy, Cairo University. Five children did not meet the inclusion criteria and the study was conducted on the remaining 45 children ( 24 boys and 21girls) who met the following inclusion criteria. Their ages ranged from 11-13 years. They had mild degree of spasticity $<2$, according to the Modified Ashworth Scale (MAS) [16]. Their level of gross motor function ranged from I to II, according to the Gross Motor Function Classification System (GMFCS) [17]. Moreover, all children had normal vision, as well as hearing and were able to follow instructions and understand testing and treatment procedures. Children were excluded from this study if they had fixed contractures or deformities of the spine or extremities, history of surgical interference of the spine or lower extremities within 1 year, auditory or visual agnosia or marked language deficits or seizure, exercise induced asthma, congenital heart defects, and cognitive impairment affecting their ability to follow instructions during assessment and training. Additionally, they were excluded if they received botulinum toxin injection within the last 6 months. Fig. 1 shows the recruitment process and flow chart of the study design.

All assessment and treatment procedures, purposes and benefits of the study were explained to all children and 


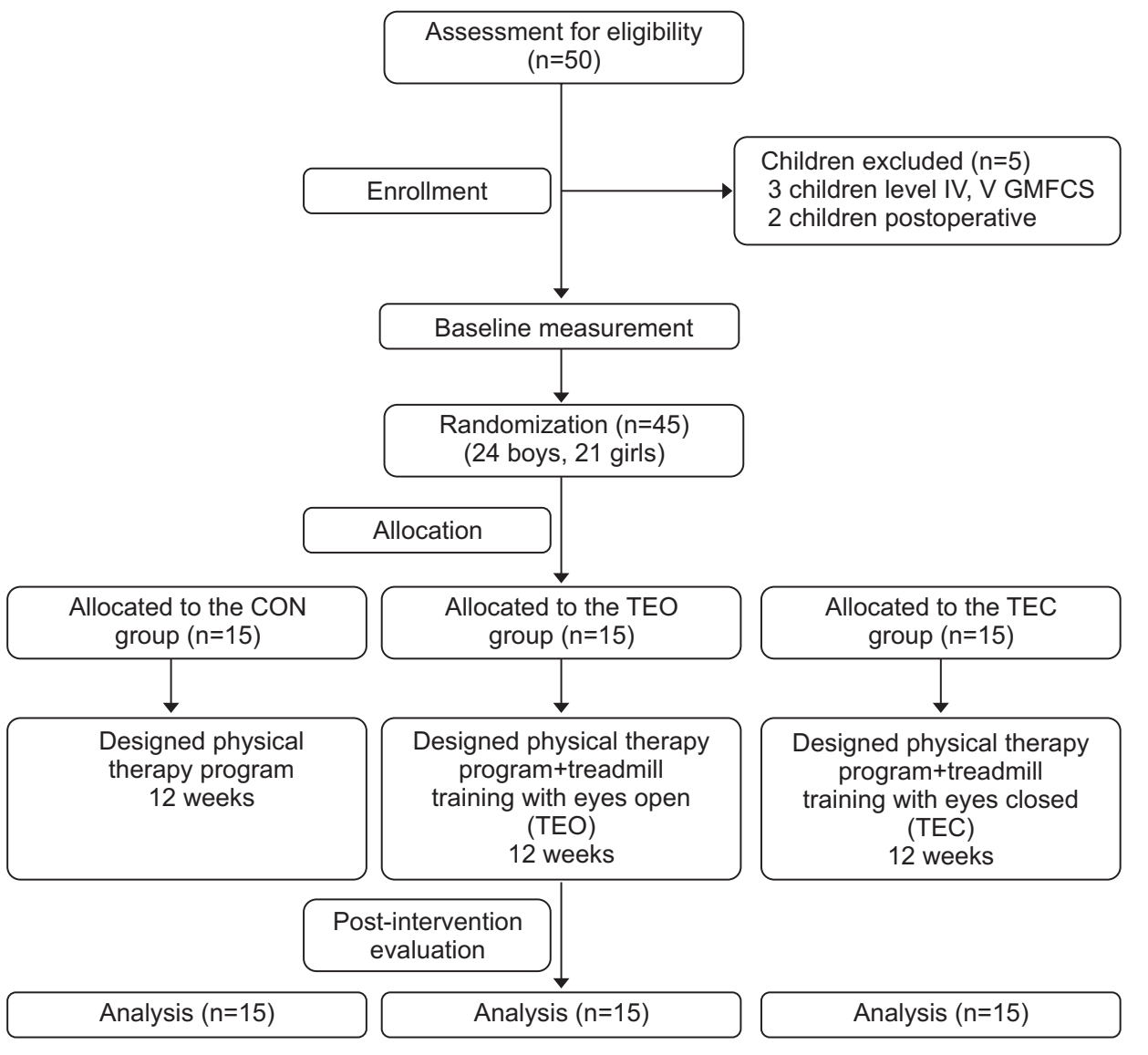

Fig. 1. Flow chart of the study design. CON, control; TEO, treadmill training with eyes open; TEC, treadmill training with eyes closed. their parents. Each child's parents signed an informed consent form indicating agreement to their child's participation before initiation of the study. The study was performed according to the Code of Ethics of the World Medical Association (Declaration of Helsinki) for experiments involving humans. This randomized controlled study was approved by the Ethics Review Committee of the Faculty of Physical Therapy, Cairo University (No. P.T.REC/012/001889).

After fulfilling the eligibility criteria and undergoing the initial assessment, the recruited children were randomly allocated into 3 groups with 15 children each by using a computer generated random number list. Control group (CON) underwent especially designed physical therapy program. Study groups (TEO and TEC) underwent the same program in addition to treadmill gait training with eyes open and closed, respectively.

\section{Outcome measures}

All children completed the following assessments at baseline (pre-treatment) and at the end of 3 succes- sive months of treatment (post-treatment). Evaluation was conducted by the assessor blinded to participants' allocation, and was not involved in the application of treatment. Knee JPS was assessed by Biodex III isokinetic dynamometer. Pediatric Balance Scale (PBS) was used to assess functional balance, while functional mobility was assessed using modified Timed Up and Go Test (mTUG).

\section{Procedures}

\section{Assessment of knee joint position sense}

Absolute degree of knee joint position error was assessed, using Biodex III isokinetic dynamometer (Biodex Medical System Inc., Shirley, NY, USA). The active repositioning test was performed to examine the ability of each child to actively reproduce a preselected joint position, at a preset angle of $45^{\circ}$ of knee flexion. It was recorded in absolute degrees of error between preselected angle and repositioned angle. Angular speed for the active reposition test was set at $2^{\circ} / \mathrm{s}$ [14].

Repositioning accuracy test was assessed for only the right lower extremity (dominant limb), to facilitate the 
testing setup. Each child was instructed to sit on the associated chair of the Biodex System with the knee of the tested extremity aligned with the axis of the dynamometer, and positioned in $90^{\circ}$ flexion (the starting position). Each child was stabilized by straps around the trunk, pelvis, and thigh. The tibial pad was secured to the shank 3 $\mathrm{cm}$ proximal to the lateral malleolus, according to manufacturer's guidelines [18].

Before being tested, every child underwent a practice session with subsequent rest period of 30 seconds. During the practice session the tested extremity was permitted to passively move to the target angle $45^{\circ}$ towards knee extension, and then, it was held for 10 seconds to enable every child to remember the test position with eyes open, then when blindfolded. During the active reposition test session, each child was blindfolded to exclude participation of visual inputs to joint repositioning requiring somatosensory input, for limb guidance to accomplish the task [7]. Each child was asked to actively move his/ her lower leg to the pre-selected test position within 60 seconds. When he/she felt that the target angle had been reached actively, he/she informed the therapist who stopped the device using the hold/release button. The leg was then returned to the starting position by the device, where it was left for 30 seconds before the next test trial. The test was repeated three times, and the average was calculated and used for statistical analysis.

\section{Assessment of functional balance}

Functional balance was assessed using PBS. It is a valid scale for measuring functional balance abilities in children with spastic CP [19]. High test-retest and interrater reliability of the PBS have been established [20]. It includes 14 items resembling different daily living activities, with a maximal score of 56 points. Tasks are scored on a 5-point ordinal scale from zero to 4 , with zero indicating failure to perform the task independently, and 4 indicating capability to perform the task without assistance. Scores are given according to duration that a position can be sustained for, distance which is reached by the upper limb in front of the body, and duration that is required for achieving the task [15].

\section{Assessment of functional mobility}

Functional mobility was assessed using mTUG. It measures functional mobility and balance, according to time required for the child to perform the task of standing from the sitting position on chair without arm supports, walking $3 \mathrm{~m}$, turning around, and returning back to the sitting position. Intra-rater and inter-rater reliability of the mTUG test are reported to be very high [21]. The time required to accomplish the task is recorded in seconds, using a stopwatch. Thereafter, the test was performed three times, and the mean was taken and used for statistical analysis.

\section{Procedures for treatment}

Treadmill gait training program: Children in both study groups underwent treadmill gait training by using Biodex Gait Trainer 2. It is used to train walking ability in individuals with neurologic gait dysfunctions [22]. Treadmill gait training protocol was conducted 3 times/week for 12 successive weeks. The training session lasted for 30 minutes, subdivided into 3 sets with 10 minutes each. Prior to treadmill gait training, children were allowed to walk on the gait trainer for 5 minutes to determine maximum walking speed accomplished by each child. During the first and last 5 minutes of the training session, every child walked at $60 \%$ maximal speed while during the remaining 20 minutes, they walked at $80 \%$ maximal speed $[23,24]$. Children in both study groups were offered a rest period for 5 minutes, after each set of training to prevent fatigue. Children in the TEO group conducted treadmill gait training with their eyes open, while the TEC group performed treadmill gait training with their eyes closed by an eye cover, according to the technique recommended by Zanetti and Schieppati [25]. All children were permitted to grasp treadmill handrails to further advance security. Additionally, verbal guidance and manual help were applied, to prevent the child from falling and to achieve better gait pattern.

Physical therapy treatment program: All children in the 3 groups underwent especially designed physical therapy program for 1 hour, 3 times/week, for 12 successive weeks. Each exercise was practiced for 5 minutes with a rest period of 2 minutes between exercises. Selected therapeutic exercises program included the following. (1) Inhibition of undesired movement pattern, and facilitation of desired movement pattern based on neurodevelopmental technique. (2) Proprioceptive training through weight-bearing activities from different positions (e.g., quadruped, kneeling, half kneeling, and standing). 
(3) Facilitation of balance reactions from different positions on balance board shifting weight forward, backward, and sideward. (4) Facilitation of standing on one leg alternatively. (5) Facilitation of protective reactions in different directions. (6) Facilitation of counterpoising mechanism through instructing the child to kick the ball from standing position, and throw the ball by his/her hands. (7) Facilitation of rising mechanism from different positions. (8) Stretching exercises for hip flexors, adductors, hamstrings, and planter flexors. (9) Strengthening exercises for back and abdominal muscles, as well as upper and lower limb muscles. And (10) Gait training activities including walking on balance beam, walking using different obstacles (e.g. stepper, wedges, and rolls), and walking up and down stairs.

\section{Statistical analysis}

Data were statistically analyzed by using SPSS version 20 (SPSS Inc., Chicago, IL, USA). Descriptive statistics (mean and standard deviation) were computed, for all measured outcomes. Analysis of variance (ANOVA) was conducted to compare mean values of parametric variables (JPS and mTUG) between the three groups before and after treatment using post-hoc test for pair wise comparison. Paired t-test was used to compare mean values within each group over intervention. Additionally, Kruskal-Wallis test was used to compare mean values of nonparametric variables (PBS), between the three groups before and after treatment. Furthermore, comparisons of mean differences between groups, and within each group were conducted using Mann-Whitney U test, and Wilcoxon signed-rank test, respectively. Statistical significance was set at $\mathrm{p}<0.05$.

\section{RESULTS}

Forty-five children with spastic diplegia were included in this study. They were randomly assigned to the CON,

Table 1. Demographic characteristics and baseline measures of the participating children

\begin{tabular}{lcccc}
\hline & CON group $(\mathbf{n}=\mathbf{1 5})$ & TEO group $(\mathbf{n}=\mathbf{1 5})$ & TEC group $(\mathbf{n}=\mathbf{1 5})$ & p-value \\
\hline Age $(\mathrm{yr})$ & $12.13 \pm 0.74$ & $11.93 \pm 0.88$ & $12.2 \pm 0.77$ & 0.64 \\
\hline Weight $(\mathrm{kg})$ & $38.4 \pm 2.38$ & $38.73 \pm 2.54$ & $37.93 \pm 2.91$ & 0.71 \\
Height $(\mathrm{cm})$ & $138.87 \pm 2.94$ & $139.53 \pm 3.11$ & $140.2 \pm 3.66$ & 0.53 \\
Gender & & & & \\
$\quad$ Male & $8(53.3)$ & $9(60)$ & $7(46.7)$ & \\
\hline Female & $7(46.7)$ & $6(40)$ & $8(53.3)$ & \\
\hline JPS $\left(^{\circ}\right)$ & $11.93 \pm 3.41$ & $12.66 \pm 3.77$ & $12.33 \pm 3.67$ & 0.858 \\
\hline PBS (score) & $39.26 \pm 3.71$ & $38.53 \pm 4.65$ & $38.73 \pm 4.31$ & 0.855 \\
\hline mTUG (s) & $21.13 \pm 3.11$ & $20.86 \pm 3.56$ & $21.46 \pm 2.44$ & 0.867 \\
\hline
\end{tabular}

Values are presented as mean \pm standard deviation or number of cases (\%).

CON, control; TEO, treadmill training with eyes open; TEC, treadmill training with eyes closed; JPS, joint position sense; PBS, Pediatric Balance Scale score; mTUG, modified Timed Up and Go test.

Table 2. Comparison of the outcome measures within each group

\begin{tabular}{|c|c|c|c|c|c|c|c|c|c|}
\hline & \multicolumn{2}{|c|}{ CON group $(n=15)$} & \multirow{2}{*}{ p-value } & \multicolumn{2}{|c|}{ TEO group $(n=15)$} & \multirow{2}{*}{ p-value } & \multicolumn{2}{|c|}{ TEC group $(n=15)$} & \multirow{2}{*}{ p-value } \\
\hline & Pre & Post & & Pre & Post & & Pre & Post & \\
\hline $\operatorname{JPS}\left({ }^{\circ}\right)$ & $11.93 \pm 3.41$ & $11.6 \pm 3.71$ & & $12.66 \pm 3.77$ & $8.53 \pm 3.50$ & $0.000^{\mathrm{a}) *}$ & $12.33 \pm 3.67$ & $5.60 \pm 2.22$ & $0.000^{\mathrm{a}) *}$ \\
\hline BS (score) & $39.26 \pm 3.71$ & $40.8 \pm 4.44$ & $0.004^{\mathrm{b}) *}$ & $38.53 \pm 4.65$ & $44.93 \pm 3.93$ & $0.001^{\mathrm{b}) *}$ & $38.73 \pm 4.31$ & $48.66 \pm 3.67$ & $0.001^{\mathrm{b}) *}$ \\
\hline TTUG (s) & $21.13 \pm 3.11$ & $19.2 \pm 2.80$ & $0.000^{\mathrm{a}) *}$ & $20.86 \pm 3.56$ & $16.46 \pm 3.02$ & $0.000^{\mathrm{a}) *}$ & $21.46 \pm 2.44$ & $13.80 \pm 2.95$ & $0.000^{\mathrm{a}) *}$ \\
\hline
\end{tabular}

Values are presented as mean \pm standard deviation.

CON, control; TEO, treadmill training with eyes open; TEC, treadmill training with eyes closed; JPS, joint position sense; PBS, Pediatric Balance Scale score; mTUG, modified Timed Up and Go test.

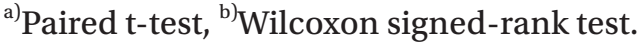

${ }^{*} \mathrm{p}<0.05$. 
TEO, and TEC groups. The three groups were homogenous at baseline with regard to age, weight, and height ( $p>0.05$ ) (Table 1). There were no significant differences between the three groups in baseline outcome measures including knee JPS, PBS, and mTUG ( $p>0.05$ ) (Table 1).

In both study groups (TEO and TEC), there was statistically significant decrease in absolute degrees of error of knee JPS between baseline, and the end of treatment $(\mathrm{p}<0.05)$ while the CON group did not significantly change after intervention ( $\mathrm{p}=0.136)$ (Table 2). Moreover, the three groups experienced statistically significant improvements in PBS over intervention $(\mathrm{p}<0.05)$ (Table 2). According to mTUG, statistically significant improvements were observed in the three groups, from pre- to post-treatment $(\mathrm{p}<0.05)$ (Table 2$)$.

Comparisons between the three groups indicated statistically significant differences in all outcome measures, after treatment between CON and TEO groups ( $p=0.01$ ), CON and TEC groups $(\mathrm{p}<0.001)$, and TEO and TEC groups ( $\mathrm{p}=0.01$ ) with greater improvements in favor of the TEC group (Tables 3, 4).

\section{DISCUSSION}

The purpose of this study was to examine the effect of treadmill training with eyes open and closed on the knee JPS, functional balance and mobility in children with spastic diplegic CP. Choosing the age of participating children to be 11-13, is in agreement with Stanley et al. [26] who affirmed that children aged 7-10 can resolve sensory conflict from somatosensory and visual receptors, and properly use the vestibular framework as a reference.

According to the results of this study, both gait training protocols either with eyes open or with eyes closed in- duced within group improvement in knee joint proprioception, functional balance and mobility with greater improvement in the TEC group. Furthermore, in the CON group, there was significant improvement in functional balance and mobility, without significant change in proprioceptive acuity of the knee joint. Therefore, these findings demonstrated that treadmill training with blocked vision could be useful, for enhancing the awareness of knee joint position, functional balance and mobility in children with spastic diplegia.

This study revealed that all groups displayed significant improvement in the measured outcomes with the exception of JPS in the CON group. This could be due to the impact of selected exercises program expected to enhance the volitional control of affected lower limbs and trunk, encourage normal movement pattern of postural control, and adjust balance and flexibility of muscles. This agrees with findings of Dodd et al. [27] who concluded that activities and rehabilitation programs improve physical capacity and functional abilities of children with CP.

Table 3. Comparison of the post-treatment outcome measures between groups

\begin{tabular}{lccrc}
\hline & $\begin{array}{c}\text { CON group } \\
(\mathbf{n}=15)\end{array}$ & $\begin{array}{c}\text { TEC group } \\
(\mathbf{n}=15)\end{array}$ & $\begin{array}{c}\text { TEO group } \\
(\mathbf{n}=15)\end{array}$ & $\begin{array}{c}\text { p- } \\
\text { value }\end{array}$ \\
\hline JPS $\left(^{\circ}\right)$ & $11.6 \pm 3.71$ & $8.53 \pm 3.5$ & $5.6 \pm 2.22$ & $0.000^{\mathrm{a}) *}$ \\
PBS (score) & $40.8 \pm 4.44$ & $44.93 \pm 3.93$ & $48.66 \pm 3.67$ & $0.000^{\mathrm{b}) *}$ \\
mTUG (s) & $19.2 \pm 2.8$ & $16.46 \pm 3.02$ & $13.8 \pm 2.95$ & $0.000^{\mathrm{a}) *}$ \\
\hline
\end{tabular}

Values are presented as mean \pm standard deviation.

CON, control; TEO, treadmill training with eyes open; TEC, treadmill training with eyes closed; JPS, joint position sense; PBS, Pediatric Balance Scale score; mTUG, modified Timed Up and Go test.

${ }^{\text {a) }}$ One-way ANOVA, ${ }^{\text {b) }}$ Kruskal-Wallis test. ${ }^{*} \mathrm{p}<0.05$.

Table 4. Pair wise comparisons of the outcome measures after intervention

\begin{tabular}{|c|c|c|c|c|c|c|}
\hline & \multicolumn{2}{|c|}{ CON vs. TEO } & \multicolumn{2}{|c|}{ CON vs. TEC } & \multicolumn{2}{|c|}{ TEO vs. TEC } \\
\hline & Mean difference & p-value & Mean difference & p-value & Mean difference & p-value \\
\hline $\operatorname{JPS}\left(^{\circ}\right)$ & 3.06 & $0.01^{\mathrm{a}) *}$ & 6 & $0.000^{\mathrm{a}) *}$ & 2.93 & $0.01^{\mathrm{a}) *}$ \\
\hline PBS (score) & 4.13 & $0.01^{\mathrm{b}) *}$ & 7.86 & $0.000^{\mathrm{b}) *}$ & 3.73 & $0.01^{\mathrm{b}) *}$ \\
\hline mTUG (s) & 2.73 & $0.01^{\mathrm{a}) *}$ & 5.4 & $0.000^{\mathrm{a}) *}$ & 2.66 & $0.01^{\mathrm{a}) *}$ \\
\hline
\end{tabular}

CON, control; TEO, treadmill training with eyes open; TEC, treadmill training with eyes closed; JPS, joint position sense; PBS, Pediatric Balance Scale score; mTUG, modified Timed Up and Go test.

${ }^{a)}$ Post-hoc test for pair wise comparison, ${ }^{\text {b) }}$ Mann-Whitney U test.

${ }^{*} \mathrm{p}<0.05$. 
Furthermore, improvement of postural control during locomotion enables the child to use proper frameworks to advance postural stability and control [28].

Findings of this study support the use of both treadmill training protocols for reducing knee joint proprioceptive error and enhancing functional balance and locomotive abilities. This could be clarified by the study of Dimitrijevic et al. [29] and Mattern-Baxter [24] who reported that treadmill training permits specific gait training in a rhythmic fashion, that stimulates central pattern generators (CPGs) in the spinal cord at the lumbar level. They added that CPGs are neural activations that enhance motor patterns and biomechanical elements included in the gait cycle, leading to rhythmic reciprocation and better postural control and balance. Furthermore, it was reported that walking training using a treadmill is more efficient than conventional walking training, which could clarify greater improvement of all measured variables in both study groups, than the control group [30].

Improvement of outcome measures in the TEO group is supported by findings of Liao and Hwang [10] who reported that visual information is essential for gross motor function in children with CP. These findings recommended that children with CP use visual cues as a compensatory system, for activities including the JPS. During visuomotor tasks, vision prevails over proprioception, particularly if the other two systems conflict [31].

Furthermore, treadmill training with allowed vision motivates children to keep postural control, and symmetric weight support. These procedures may improve knee joint proprioception and functional balance and mobility, by appropriately incorporating somatosensory, vestibular, and visual stimulation. These findings also could be explained by the study of Ledebt et al. [32] who concluded that balance training with visual input is useful in diminishing postural sway, and improves voluntary weight shift during standing.

The mechanism for improvement of the knee JPS, balance and locomotive abilities in the TEC group is probably linked to additional activation of joint and muscle receptors accomplished by visual restriction which imposed the children to use somatosensory and vestibular senses to walk and balance, by restricting excessive visual reliance. Within the same context, it was reported that treadmill gait training with blocked vision, is effective at recovering gait, balance abilities, and proprioceptive acuity in individuals with stroke through improvement of postural control elicited by enhancing proprioceptive and vestibular sensations during visual restriction [33,34].

Walking with visual deprivation reinforces neural associations that emerge from participation of cortical structures included in the locomotion imagination, foot positioning, and dynamic postural control. It enhances awareness of body position image in relation to surroundings. This could be due to impact of motor imagery which plays a fundamental role during blindfolded walking [35]. Moreover, Rodrigues et al. [36] found that kinesthetic motor imagery activates motor representations engaged with postural control. Therefore, kinesthetic motor imagery, more than visual motor imagery, can adjust body sway during balance control.

Additionally, Hallemans et al. [37] reported that people assume different gait patterns while walking blindfolded. When sighted people walk with blocked vision, they tend to use an attentive gait pattern towards optimal step frequency, to minimize step errors during walking. Therefore, state estimation is used to precisely control foot position, which appears to depend on modifying weighting of sensory prediction errors [38].

Furthermore, these findings are supported by Zanetti and Schieppati [25] who reported that treadmill practice with visual restriction performed by healthy adults enhanced their balance capability in comparison to a vision-allowed group. Goble et al. [39] reported that repeated stimulation of somatosensory receptors in children with hemiplegic CP may be accountable for improving their balance abilities through proprioceptive reintegration.

Significant differences between groups post-training in favor of TEC group could be explained by the study of Oliveira et al. [40] who found higher neural processing identified with motor planning and execution, when vision is not accessible during locomotion. They added that walking with blocked vision, influences attention and cognitive abilities. Therefore, the necessity for attention/ cognitive demands may affect electrocortical changes at somatosensory cortical areas, as well as prefrontal and premotor areas achieving a noteworthy improvement in dynamic stability during gait.

Some limitations of this study warrant consideration including the small sample size which makes it difficult to generalize results, and absence of follow-up after in- 
tervention did not allow for long term effect of therapy to be determined. Accordingly, further larger-sample studies with long-term follow-up are required. Further studies are needed to better understand how blocked vision treadmill gait training, could affect kinematic and kinetic gait parameters in children with CP.

In conclusion, this study demonstrated that both treadmill training protocols are effective in improving knee joint proprioceptive sense, functional balance, and locomotion in children with spastic diplegia. Moreover, treadmill training with blocked vision is recommended, when planning a gait rehabilitation program for children with $\mathrm{CP}$ to take advantage of adopting alternative sensory strategies relative to adjusting joint proprioception, balance activities, and mobility without the help of vision.

\section{CONFLICT OF INTEREST}

No potential conflict of interest relevant to this article was reported.

\section{ACKNOWLEDGMENTS}

The author would like to thank all the children and their parents who participated and collaborated in this study.

\section{REFERENCES}

1. Saavedra S, Woollacott M, van Donkelaar P. Head stability during quiet sitting in children with cerebral palsy: effect of vision and trunk support. Exp Brain Res 2010;201:13-23.

2. Peterka RJ. Sensorimotor integration in human postural control. J Neurophysiol 2002;88:1097-118.

3. Hallemans A, Ortibus E, Meire F, Aerts P. Low vision affects dynamic stability of gait. Gait Posture 2010;32:547-51.

4. Takakusaki K. Functional neuroanatomy for posture and gait control. J Mov Disord 2017;10:1-17.

5. Rossignol S, Dubuc R, Gossard JP. Dynamic sensorimotor interactions in locomotion. Physiol Rev 2006;86:89-154.

6. Damiano DL, Wingert JR, Stanley CJ, Curatalo L. Contribution of hip joint proprioception to static and dynamic balance in cerebral palsy: a case control study.
J Neuroeng Rehabil 2013;10:57.

7. Wingert JR, Burton H, Sinclair RJ, Brunstrom JE, Damiano DL. Joint-position sense and kinesthesia in cerebral palsy. Arch Phys Med Rehabil 2009;90:447-53.

8. Lieber RL, Friden J. Spasticity causes a fundamental rearrangement of muscle-joint interaction. Muscle Nerve 2002;25:265-70.

9. Rose J, Wolff DR, Jones VK, Bloch DA, Oehlert JW, Gamble JG. Postural balance in children with cerebral palsy. Dev Med Child Neurol 2002;44:58-63.

10. Liao HF, Hwang AW. Relations of balance function and gross motor ability for children with cerebral palsy. Percept Mot Skills 2003;96(3 Pt 2):1173-84.

11. Girolami GL, Shiratori T, Aruin AS. Anticipatory postural adjustments in children with hemiplegia and diplegia. J Electromyogr Kinesiol 2011;21:988-97.

12. Smania N, Bonetti P, Gandolfi M, Cosentino A, Waldner A, Hesse S, et al. Improved gait after repetitive locomotor training in children with cerebral palsy. Am J Phys Med Rehabil 2011;90:137-49.

13. Grecco LA, Tomita SM, Christovao TC, Pasini H, Sampaio LM, Oliveira CS. Effect of treadmill gait training on static and functional balance in children with cerebral palsy: a randomized controlled trial. Braz J Phys Ther 2013;17:17-23.

14. Panchal MD, Joshua AM, Tedla JS. Comparison of knee joint proprioception in spastic diplegic and typically developing children of age 5-12 years: a cross sectional study. Int J Curr Res Rev 2011;3:228-34.

15. Kembhavi G, Darrah J, Magill-Evans J, Loomis J. Using the berg balance scale to distinguish balance abilities in children with cerebral palsy. Pediatr Phys Ther 2002;14:92-9.

16. Bohannon RW, Smith MB. Interrater reliability of a modified Ashworth scale of muscle spasticity. Phys Ther 1987;67:206-7.

17. Palisano RJ, Cameron D, Rosenbaum PL, Walter SD, Russell D. Stability of the gross motor function classification system. Dev Med Child Neurol 2006;48:424-8.

18. Wilk K. Isokinetic testing: setup and positioning. In: Biodex System II Manual: Applications/Operations. New York: Biodex System Inc.; 1991. http://www.biodex. com/sites/default/files/s2s_apman_90317.pdf.

19. Yi SH, Hwang JH, Kim SJ, Kwon JY. Validity of pediatric balance scales in children with spastic cerebral palsy. Neuropediatrics 2012;43:307-13. 
20. Her JG, Woo JH, Ko J. Reliability of the pediatric balance scale in the assessment of the children with cerebral palsy. J Phys Ther Sci 2012;24:301-5.

21. Dhote SN, Khatri PA, Ganvir SS. Reliability of "Modified timed up and go" test in children with cerebral palsy. J Pediatr Neurosci 2012;7:96-100.

22. Tong RK, Ng MF, Li LS. Effectiveness of gait training using an electromechanical gait trainer, with and without functional electric stimulation, in subacute stroke: a randomized controlled trial. Arch Phys Med Rehabil 2006;87:1298-304.

23. Damiano DL, DeJong SL. A systematic review of the effectiveness of treadmill training and body weight support in pediatric rehabilitation. J Neurol Phys Ther 2009;33:27-44.

24. Mattern-Baxter K. Locomotor treadmill training for children with cerebral palsy. Orthop Nurs 2010;29:169-73.

25. Zanetti C, Schieppati M. Quiet stance control is affected by prior treadmill but not overground locomotion. Eur J Appl Physiol 2007;100:331-9.

26. Stanley F, Blair E, Alberman E. Cerebral palsies: epidemiology and causal pathways. London: Mac Keith Press; 2000.

27. Dodd KJ, Taylor NF, Graham HK. A randomized clinical trial of strength training in young people with cerebral palsy. Dev Med Child Neurol 2003;45:652-7.

28. Kamm K, Thelen E, Jensen JL. A dynamical systems approach to motor development. Phys Ther 1990;70:76375.

29. Dimitrijevic MR, Gerasimenko Y, Pinter MM. Evidence for a spinal central pattern generator in humans. Ann N Y Acad Sci 1998;860:360-76.

30. Willoughby KL, Dodd KJ, Shields N, Foley S. Efficacy of partial body weight-supported treadmill training compared with overground walking practice for children with cerebral palsy: a randomized controlled trial. Arch Phys Med Rehabil 2010;91:333-9.
31. Jones KE, Wessberg J, Vallbo A. Proprioceptive feedback is reduced during adaptation to a visuomotor transformation: preliminary findings. Neuroreport 2001;12:4029-33.

32. Ledebt A, Becher J, Kapper J, Rozendaalr RM, Bakker R, Leenders IC, et al. Balance training with visual feedback in children with hemiplegic cerebral palsy: effect on stance and gait. Motor Control 2005;9:45968.

33. Kim YW, Moon SJ. Effects of treadmill training with the eyes closed on gait and balance ability of chronic stroke patients. J Phys Ther Sci 2015;27:2935-8.

34. Moon SJ, Kim YW. Effect of blocked vision treadmill training on knee joint proprioception of patients with chronic stroke. J Phys Ther Sci 2015;27:897-900.

35. Bakker M, De Lange FP, Helmich RC, Scheeringa R, Bloem BR, Toni I. Cerebral correlates of motor imagery of normal and precision gait. Neuroimage 2008;41:998-1010.

36. Rodrigues EC, Lemos T, Gouvea B, Volchan E, Imbiriba LA, Vargas CD. Kinesthetic motor imagery modulates body sway. Neuroscience 2010;169:743-50.

37. Hallemans A, Beccu S, Van Loock K, Ortibus E, Truijen S, Aerts P. Visual deprivation leads to gait adaptations that are age- and context-specific: II. Kinematic parameters. Gait Posture 2009;30:307-11.

38. Maeda RS, O'Connor SM, Donelan JM, Marigold DS. Foot placement relies on state estimation during visually guided walking. J Neurophysiol 2017;117:480-491.

39. Goble DJ, Hurvitz EA, Brown SH. Deficits in the ability to use proprioceptive feedback in children with hemiplegic cerebral palsy. Int J Rehabil Res 2009;32:267-9.

40. Oliveira AS, Schlink BR, Hairston WD, Konig P, Ferris DP. Restricted vision increases sensorimotor cortex involvement in human walking. J Neurophysiol 2017;118:1943-51. 